\title{
Vaccination of cows against mastitis-an alternative to antibiotics application
}

\author{
M. N. Isakova ${ }^{1, *}, U . V$. Sivkova $^{1}$ and M.V. Ryaposova ${ }^{1}$ \\ ${ }^{1}$ Federal State Budgetary Institution "Ural Federal Agrarian Scientific Research Center of the Ural \\ Branch of the Russian Academy of Sciences", 620142 Yekaterinburg, Russia
}

\begin{abstract}
Data demonstrating the effectiveness of the antimastitic vaccine in bred livestock farms in the Sverdlovsk region by examining the level of diagnosed mastitis and the index of somatic cells (SCC) in milk are given. Application of animal immunization against mastitis revealed that 6 months after the first vaccination of animals in the pedigree breeding unit and plant, the number of identified mastitis reduced by $16.6 \%$ and $7.3 \%$, respectively, and the level of SCC in the milk of highly productive cows by $26.5 \%$ and $10.7 \%$, respectively. 12 months after the first vaccination protocol was applied. The overall number of animals with evidence of mastitis in the pedigree breeding unit and plant was $13.3 \%$ and $7.5 \%$, respectively. The SCC level in 1 organization was 290 thousand $/ \mathrm{ml}$, and in 2 - 450 thousand / $\mathrm{ml}$. The research found a significant reduction in the diagnosed mastitis in animals after 32 months due to the application of the antimastitis vaccine. The overall number of diseases reduced by $30.6 \%$ in organization 1 and $26.2 \%$ in organization 2 comparing to the period of the beginning of vaccination. Results: the dynamics of decrease in asymptomatic and clinical mastitis in organization 2 comparing to the period at the time of immunization was $19.5 \%$ and $11.1 \%$, respectively. And in organization 1, the level of asymptomatic and clinical mastitis decreased by $16.9 \%$ and $9.0 \%$, respectively. The quantity of SCC in the milk of cows kept in the research organization 1 had a value of 152 thousand $/ \mathrm{ml}$, which is $83.9 \%$ lower comparing to the first period of the study. The level of SCC in the tested samples of milk of animals kept in organization 2 was 263 thousand $/ \mathrm{ml}$, which is $62.2 \%$ lower than this index before the application of the animal vaccination program against mastitis.
\end{abstract}

\section{Introduction}

In the current dairy farming, great significance is assigned to the issue of increasing milk production. One of the most essential conditions for this is the qualitative enhancement of the stock of breeds and the increase of their genetic capacity [1]. More and more attention is given to the quality of unpasteurized milk [2]. During the primary stage of milk production, the main factor promoting a reduction in its quality indices is the existence

\footnotetext{
*Corresponding author: nauka_sokolova@mail.ru
} 
of an inflammatory process in the lacteous gland of cows [3]. Several methods are used to control mastitis, including appropriate milking technology, conformance with hygiene requirements, and use of antibacterial treatment. During the last decade, vaccines have been extremely frequently used in control protocols $[4,5,6]$. Vaccination of highly-productive cows may be used as a preventive measure for mastitis, resulting in an improvement in the quality of milk produced $[7,8,9]$.

Objective: to measure the effectiveness of the Startvac anti-mastitis vaccine in bred livestock farms in the Sverdlovsk region.

\section{Materials and methods}

The studies were conducted during 2013-2019 in the laboratory of pathology of reproductive organs and diseases of young animals of the Ural Federal agrarian research center of the Ural Branch of the Russian Academy of Sciences. Experimental data were obtained during the entire experimental study on the ground of breeding agricultural organizations $(1,2)$ in the Sverdlovsk region. Breeding organization 1 is situated in the Beloyarsky district and contains 1,815 black-and-white cows. Milking technology is performed using "Herringbone 6x12" milking machine. Breeding organization 2 is situated in the Sysert district and contains 1,400 black-and-white cows. The complex uses the technology of milking in a special lactorium using the "Parallel" device. The average annual milk productivity of the highly-productive cows studied is more than $9000 \mathrm{~kg}$.

Early studies have revealed that Staphylococcus aureus $(29.41 \%)$ and Streptococcus spp. $(23.53 \%)$ were the main microorganisms detected in samples of cow udder fluid in the farms under study. Enterococcus bacteria were seeded in $11.76 \%$ of the samples. Mold fungi of the genus Aspergillus were recorded in $17.65 \%$ of the samples. Gram-negative bacteria of the genus Klebsiella spp.(5.88\%), Pseudomonas (5.88\%), and Enterobacter $(5.88 \%)$ were also found. In this case, infectious agents of mastitis were distinguished in the monoculture $(27.3 \%)$, in the association of bacterial cultures $(55.6 \%)$, fungi and yeast $(17.1 \%)$. These studies have demonstrated that mastitis in animals with a high SCC content was triggered by a fairly wide range of pathogens. Nevertheless, a large share of cases of mastitis caused by Staphylococcus aureus, treatment of mastitis caused by Staphylococcus aureus with antimicrobial agents does not give a favorable therapeutic effect, causing a recurrent course, a predisposition to chronization, and the development of resistance to traditional antimicrobial drugs. Through prevention and avoidance of the development of antibiotic resistance, vaccination is an alternative in mastitis control protocols.

The Startvac antimastitis vaccine (Hipra, Spain) being the key component containing inactivated cells of Eschehchia coli J5 and Staphylococcus aureus CP8 strains, which have a mucosal antigen complex (SAAC) in their structure. In the studied bred livestock farms, a 3:3:3 vaccination program was used. Effectiveness of the antimastitic vaccine was assessed accounting for the level of diagnosed mastitis and the SCC index in milk. These indices were taken into account in the time period before vaccination, at the beginning of vaccination, $6,12,18,24$ and 36 months after the first vaccination. 10 vaccinations were done over a period of 32 months.

Mastitis in animals was diagnosed according to the Guidelines for the diagnosis, treatment and prevention of mastitis in cows, Guidelines for the diagnosis, treatment and prevention of asymptomatic mastitis in cows in the period of less milk production.

Asymptomatic mastitis was diagnosed using the rapid diagnostic test "Kenotest" (CID LINES, Belgium).

The level of SCC in cow's milk was defined in the laboratory of selection control of milk quality of the Regional information and selection center (RISC) of "Uralplemcenter" 
OOO, situated in Yekaterinburg. This laboratory utilized equipment from Bentley Instruments (USA), which included the Bentley-150 analyzer and the SCC Somacount-150 counter. Additionally, a monthly survey of combined unpasteurized milk for the SCC index was carried out using the DCC analyzer from DeLaval (Sweden). In total, 82039 samples were analyzed.

\section{Research results}

Studies performed prior to the start of the program of vaccination of highly-productive cows against mastitis in bred livestock farms of the Sverdlovsk region revealed a high percentage of inflammatory diseases of the lacteous gland.

During animal immunization, the amount of diagnosed mastitis in breeding organization 1 was $35 \%$, the largest number of animals were diagnosed with asymptomatic form of mastitis $(23.0 \%)$, and the clinical form was found in $12.0 \%$ of the cows studied. The mean amount of SCC in milk was 947 thousand $/ \mathrm{ml}$.

6 months after the first vaccination of animals against mastitis, the level of lacteous gland inflammatory disorders slowly reduced. The number of diagnosed mastitis reduced by $16.6 \%$ and amounted to $18.4 \%$, while the number of asymptomatic and clinical forms of the disease was at the level of $15.0 \%$ and $3.4 \%$, respectively. The SCC index in the milk of highly-productive cows reduced by $26.5 \%$ and amounted to 251 thousand $/ \mathrm{ml}$.

12 months after the application of the first Startvac anti-mastitis vaccine protocol, the tendency of a steady decrease in the level of diagnosed mastitis continued. The total number of animals with signs of mastitis was $13.3 \%$, which is $5.0 \%$ lower compared to the preceding period of the study. The level of latent inflammation in the udder was $11.2 \%$, and the number of animals with clinical manifestations of mastitis was $2.1 \%$, which is $3.8 \%$ and $1.3 \%$ lower than these indices six months after immunization. Over this period of the research, we noticed a slight increase in the level of SCC in milk samples up to 290 thousand $/ \mathrm{ml}$, which may relate to minor errors in milking technology and (or) changes in the way animals are kept and fed, fluctuations in weather conditions, and the probability of predisposing diseases.

During the study performed after 24 months, the level of diagnosed mastitis was at the level of $6.0 \%$ of the total studied number of highly-productive cows. In the structure of mastitis, the proportion of asymptomatic and clinical forms accounted for $5.6 \%$ and $0.4 \%$ of highly-productive cows, respectively (figure 1). The average range of SCC in milk reduced by 80 thousand / $\mathrm{ml}$ and amounted to 210 thousand / $\mathrm{ml}$. 


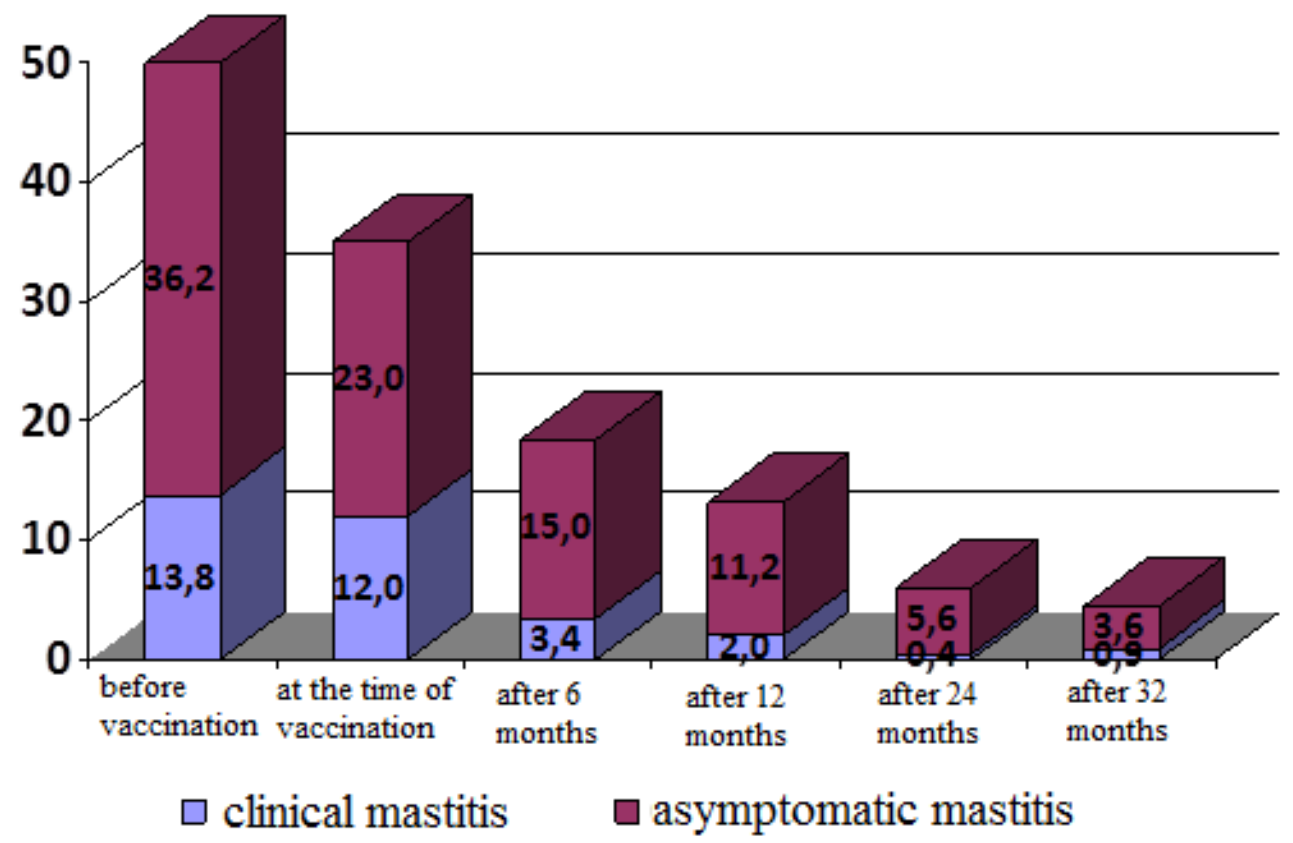

Fig. 1. The level of mastitis in the breeding organization 1 against the background of the "Startvac" vaccine», $\%$

Within 32 months, the study found a significant reduction in the diagnosed mastitis in animals against the background of the use of the Startvac anti-mastitis vaccine. The total amount of the disease reduced by $30.6 \%$ compared to the period of the beginning of vaccination, and amounted to $4.4 \%$ of the animals from the total herd. The amount of asymptomatic and clinical inflammation in the lacteous gland was in the range of $3.5 \%$ and $0.9 \%$, respectively. As a result, the dynamics of reducing these indices compared to the period at the time of immunization was $19.5 \%$ and $11.1 \%$, respectively (figure 1 ). The amount of SCC in animal milk was 152 thousand $/ \mathrm{ml}$, which confirmed a steady decline in his index by $83.9 \%$ comparing to the first period of the study (figure 2).

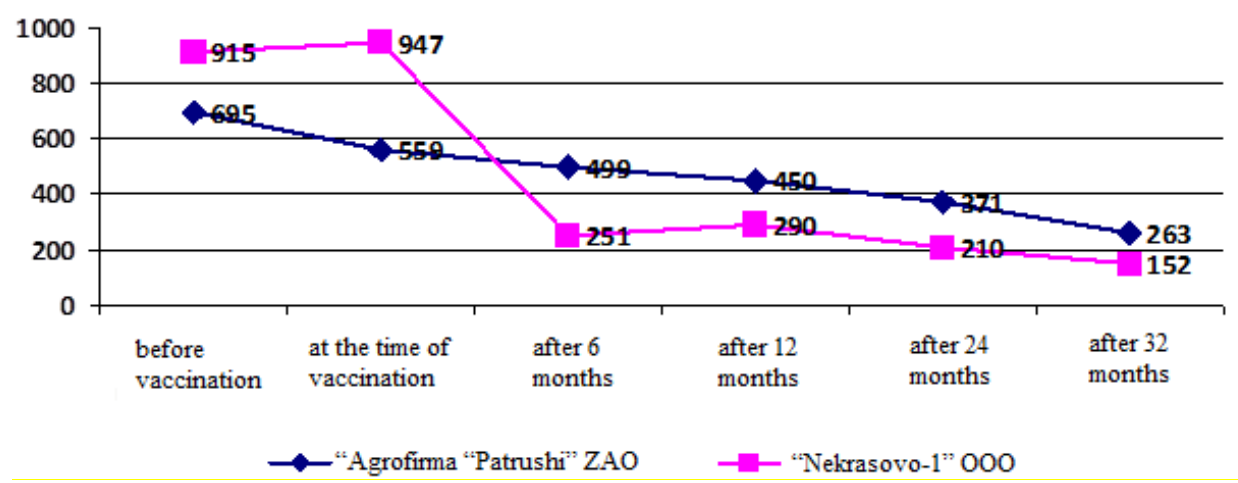

Fig. 2. Dynamics of the SCC level in cow's milk against the background of the application of antimastitic vaccine, thousand $/ \mathrm{ml}$ 
Before the application of the vaccination protocol in breeding organization 2, the level of mastitis in animals was $14.8 \%$. The most frequent asymptomatic form of mastitis was identified in animals in $11.9 \%$ of cases, and the clinical form was $2.9 \%$ of the total animal units. In the research of milk samples, the amount of SCC contained in it was 559 thousand $/ \mathrm{ml}$.

Following 6 months of the first cow immunization protocol, there is a progressive reduction in the number of identified cases of mastitis in animals. The overall level of mastitis was $7.5 \%$ (asymptomatic $-5.2 \%$, clinical $-2.3 \%$ ). The quantitative index of SCC in milk was 499 thousand / $\mathrm{ml}$, which is $10.7 \%$ thousand $/ \mathrm{ml}$ lower than this index at the time of the first vaccination.

Within 12 months, the trend towards a decline in the diagnosed mastitis in highproductive cows remains. In the research of livestock, the level of mastitis was $7.5 \%$, the proportion of asymptomatic and clinical forms of the inflammatory process in the udder accounted for $4.7 \%$ and $2.8 \%$, respectively. There is a insignificant but stable reduction in the level of SCC in the milk of highly-productive cows by 49 thousand $/ \mathrm{ml}$.

Within 24 months, there was an insignificant growth in animals with a asymptomatic form of mastitis to $5.0 \%$, while the total level of the disease remained in a downward trend and amounted to $5.7 \%$, due to the minimum number of detected clinical mastitis- $0.7 \%$ (figure 3). The SCC in milk was 371 thousand / $\mathrm{ml}$, which is 79 thousand/ml lower compared to the last study period.

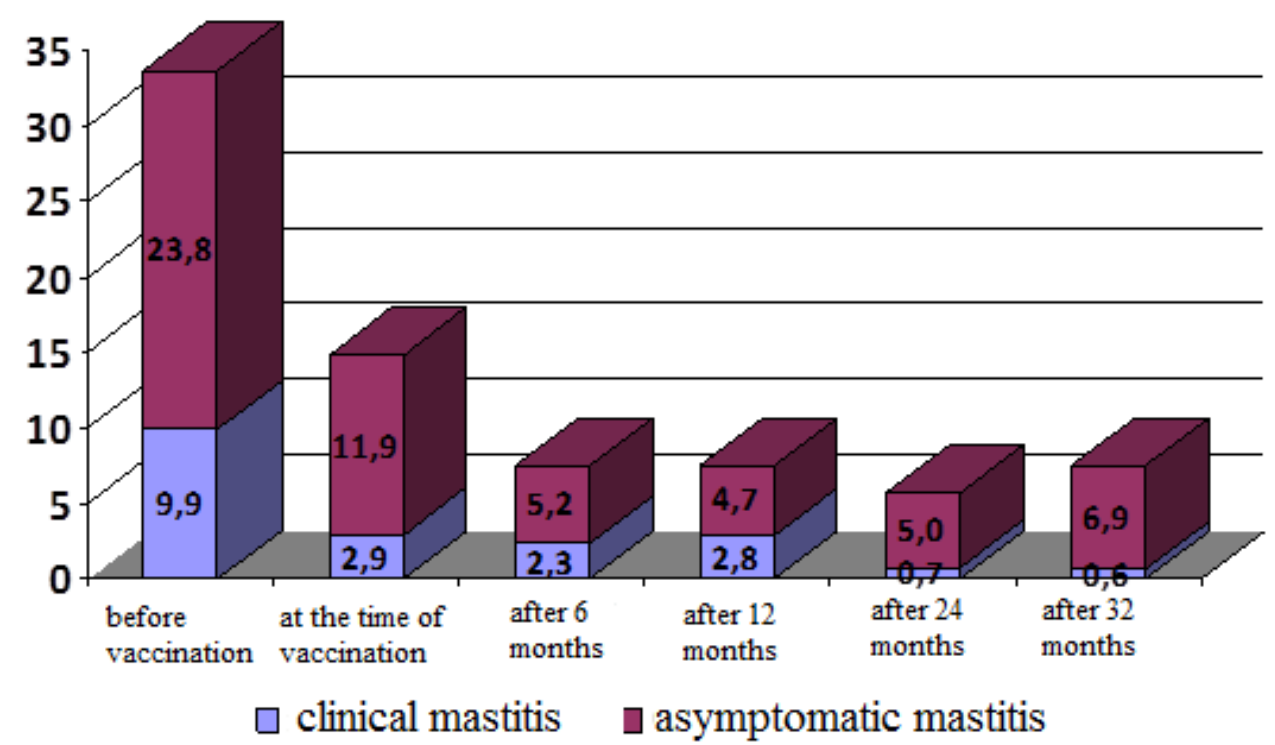

Fig. 3. The level of mastitis in the breeding organization 2 against the application of the "Startvac" vaccine», $\%$

In a study performed 32 months after using the first vaccination protocol, the total amount of animals with mastitis was $7.5 \%$. The level of asymptomatic mastitis was $6.9 \%$, which is $1.9 \%$ more than the previous study period. The level of clinical mastitis since the beginning of the first vaccination has continued its downward trend, and amounted to $0.6 \%$. Therefore, comparing these indices with the period at the time of vaccination, we observe a general decrease in the number of diagnosed mastitis by $26.2 \%$, while the number of asymptomatic forms reduced by $16.9 \%$, and the clinical form-by $9.0 \%$ (figure 3 ). The SCC 
rate in the tested milk samples was 263 thousand $/ \mathrm{ml}$, which is $62.2 \%$ lower than this index before the application of the animal vaccination protocol against mastitis (figure 2).

\section{Conclusion}

An investigation of the efficacy of the antimastitic vaccine in bred livestock farms in the Sverdlovsk region revealed a positive trend in the decrease of diagnosed mastitis in animals, as well as a decline in the level of SCC in the bulk milk of cows. Comparing the two breeding organizations, we see distinctions in the level of downturn in the studied indices, but a positive trend is seen in both farms. So in the agricultural organization 1, a reduction in the incidence of highly-productive cows with mastitis is observed during the entire analyzed time, and in the agricultural organization 2 , there is a minimal abrupt downward trend. Thus, thetotal reduction in the level of diagnosed mastitis in animals in breeding organizations after 32 months from the date of the first vaccination was $30.6 \%$ and $26.2 \%$. Therefore, the obtained findings demonstrated a positive trend in reducing the level of diagnosed mastitis and the SCC indicator in milk against the background of the use of the Startvac vaccine. Evaluation of the further impact of the antimastitis vaccine on the lacteous gland and the milk composition of highly-productive cows, as well as the protocol of application in the frame of the program for the prevention of mastitis and quality control of the collected milk, will help to further research in this direction.

\section{References}

1. I.A. Shkuratova, I.M. Donnik, A.G. Isaeva, A.S. Krivonogova. Voprosy normativnopravovogo regulirovaniya v veterinarii, 2, 366-369 (2015) (In Russian)

2. A.V. Avdeyenko, A.V. Molchanov, D.V. Krivenko, T.N. Rodionova, D.S. Markova. Vestnik Kurganskoy GSKHA, 2(18) 28-30 (2016) (In Russian)

3. S. Pepers. Veterinariya, 11, 10-13 (2018) (In Russian)

4. A. Galkin, E. Trepalina. Kombikorma, 1, 105-107 (2018) (In Russian).

5. R.A. Amanullin. Prikladnaya mikrobiologiya, 2, 1 (4), 40-44 (2015) (In Russian)

6. N.G. Vasileiou, D.C. Chatzopoulos, P.J. Cripps, K.S. Ioannidi, et al. J Dairy Sci, 102(10), 9328-9344 (2019) doi: 10.3168/jds.2019-16287. Epub 2019 Aug 7.

7. V. Bronzo. BIO, 5(212), 12-13 (2018) (In Russian).

8. B.I. Zuhair. Vet World, 10(9), 1057-1062 (2017)

9. S.B. Philip, N. Mayur, G.S. Robert . Surg Infect (Larchmt), 19(8),750-756 (2018) 